\title{
Load Testing Analyzer for Web Application
}

\author{
Shivangi Kaushal
}

\begin{abstract}
Testing is not debugging. The procedure comprising of all lifecycle exercises that fulfill determined necessities, to show that they are fit for reason and to identify the imperfections is called as Testing. To discover the mistakes and errors software testing is essential procedure. The point testing a web application comprises of implementing the application utilizing mixes of info and result to uncover failures. There are various testing procedures which serve unique purposes however it is difficult to track down and right all the inaccuracies. Software testing on an online application is accomplished with the goal that to meet the customer's requirements the performance, quality of the application remains intact. Through this paper, to analyze the load the website can carry up to which extent, the stress is made on the application part. The application is laid in a great deal of regularity frequently to shattering juncture such as the steadiness of the website maybe possibly deemed. This is completed by affixing the code of JavaScript to the application page whose tension requires to be tested. To use the results in future for further studies by the developer or the tester they are stored in a database.
\end{abstract}

KEYWORDS- Stress testing, Load Testing, Web based application, Performance testing.

\section{INTRODUCTION}

Web programs have become large (massive) in the maximum latest decade [1-3]. Web applications ought to manage countless clients with the massive expansion of the ubiquity of web applications. To give the maximum amount of the customers try the application a way to fabricate has become important to make the website simple and at ease of use. The advancement of Web applications is following an advancement like that watched for programming frameworks: A well versed structure of the workflow is maintaining the quality because the production movement is going from the craftsmanship to the industrial level [4-9]. A Web application can be taken into consideration as a disseminated framework, with a consumer server or multi-level design [10-13].

Software testing is an approach to identify mistakes in the online application. Any one particular phase of the advancement life cycle does not require software testing of

\section{Manuscript received May 20, 2020}

Shivangi Kaushal, Assistant Professor Department of Computer Science Amity University, Haryana, India (e-mail: shivangikaushal.87@gmail.com). the product however it has numerous territories for testing, for example, the entire periods of the existence cycle, for example, prerequisite detail, structure, execution, and support matters. In the beginning only the software testing is operated to discover blames and repair them. Software testing is viewed as sufficient on the off chance that it can expel the deficiencies or blunders to the most extreme degree from the online applications. Experiments are composed to approve the testing inclusion of the application. Experiments are performed and the yield is contrasted and the normal outcome. Test testimony is readied that can be saved for forthcoming reference.

The Main point of application testing comprises of achieving the application using mixes of input and state to disclose failures [2]. Faults of the humans can cause severe failures. Web application testing needs models and tools to test different parts of the application. In this work, the significance of the innovation is because of the code created in Java which is composed just a single time and might be run anyplace when connected with web-application which implies it can be reused the parts in an online application.

\section{LITERATURE SURVEY}

There are various aspects or tools used to do load and stress testing such as Apache, Jmeter, Selenium, Cucumber, Appium, TestNG and many more. the reconciliation of more apparatuses and open source libraries, the general procedure of programming testing in different measurements can be advanced on numerous parameters so the conveyance of programming item with the bug free and having better performance [14-15]. Software testing is the primary action performed for assessing item quality, and for improving it, by recognizing deserts in programming concentrated frameworks [16]. Manual testing is generally a hard and tedious and costly movement. A few investigations show that testing is considered as one of the most exorbitant advancement processes, sometimes surpassing fifty percent of absolute improvement costs [17]. Kallepalli and Tian [18] propose a measurable methodology for testing web application. Rather than breaking down Web applications legitimately, this model gathers ancient rarities, for example, control stream, information stream, outstanding burden, blunder data, and so forth., from log documents which are produced in the running of a of Website. Considering the gathered ancient rarities, this methodology may perhaps explain clients' concentration and steer the testing. The unwavering quality of the submission could be acquired from the mistake data. Moreover, the accuracy of the methodology is restricted by the log documents. Blunders that cannot be noted by log documents, for example, wrong data in created pages, 
cannot be recognized by this methodology.

\section{DIFFERENT SOFTWARE TESTING}

There are an enormous number of programming testing strategies. This paper discusses three different techniques of testing which are performance, load and stress.

\section{A. Performance Testing}

So as to get the running parameters of the Web servers and guarantee that their reacting times are adequate by clients as a rule, execution testing is expected to decide such information as the interfacing speed, load level, load intension and nonstop utilizing. This sort of testing is significant for Web applications, for in whenever, clients may go to other serious Websites because of the insufferable pausing. For this kind of testing, robotized testing instruments are expected to create the framework outstanding task at hand and assemble the exact framework execution and burden measurements under various degrees of client visiting. The typical strategy for execution testing is to arrangement various virtual clients by Scripts, which visit and associate with the Web application consequently and simultaneously, copying the meeting activities of the real clients, in order to decide the running execution parameters of this framework. Accordingly, under the precondition of decreasing the testing cost, we inquire about how to acquire the real and viable visiting activities of clients, and apply them into the age procedure for the virtual clients, in order to make the meeting impact is steady with the real factors.

\section{B. Load Testing}

During load testing, the system performance is assessed with the help of a predefined load level. While doing this, it's major objective is to calculate the time taken to perform a range of pre-defined under stipulated conditions. After doing observation on these already decided conditions, few includes as highest level of activity and minimum configuration for the ongoing web application. To perform these activity, associated data is recorded and sometimes observation on whether task are performed within the given time duration. Apart from that sometimes it also generates the logbooks on their progress. Generally, the mal-functioning of any web application is also tested by such load testing process.

\section{Stress Testing}

A framework stress test alludes to tests that lay a more prominent accentuation on vigor, accessibility and blunder dealing with under a substantial burden, instead of on what might be viewed as right conduct under typical conditions [3]. Stress testing checks the online applications three fundamental viewpoints, for example, reaction time, throughput, accessibility, and utilitarian trustworthiness. Stress testing is the point at which the online application is placed under extraordinary circumstances which meant pressure is forced upon it past ordinary environments and it arrives at the limit of pressure. The result of pressure testing generates execution, unwavering quality, protection, nature of the electronic application.

\section{PROCESSING OF STRESS TESTING}

The point of the investigation effort is to evaluate the load of the website. The web application testing apparatus, which is in real a scripting tool for the customer as a JavaScript code. Appending of the code with the website application, on which the stress testing must be completed. In this a web structure is shown which accepts the quantity of clients as the info. At that point click on the submit button. Off camera, the module that is appended with the site operates. While making a call to PageLoadTime.js, this in turn make a call to loadingtime.jsp by taking load time as an actual argument. By doing so, this procedure evaluates the load time taken by the selected website and make an entry of the same named as load time. While forming this report, three types of attribute are mentioned named opening dates, time taken and start time.

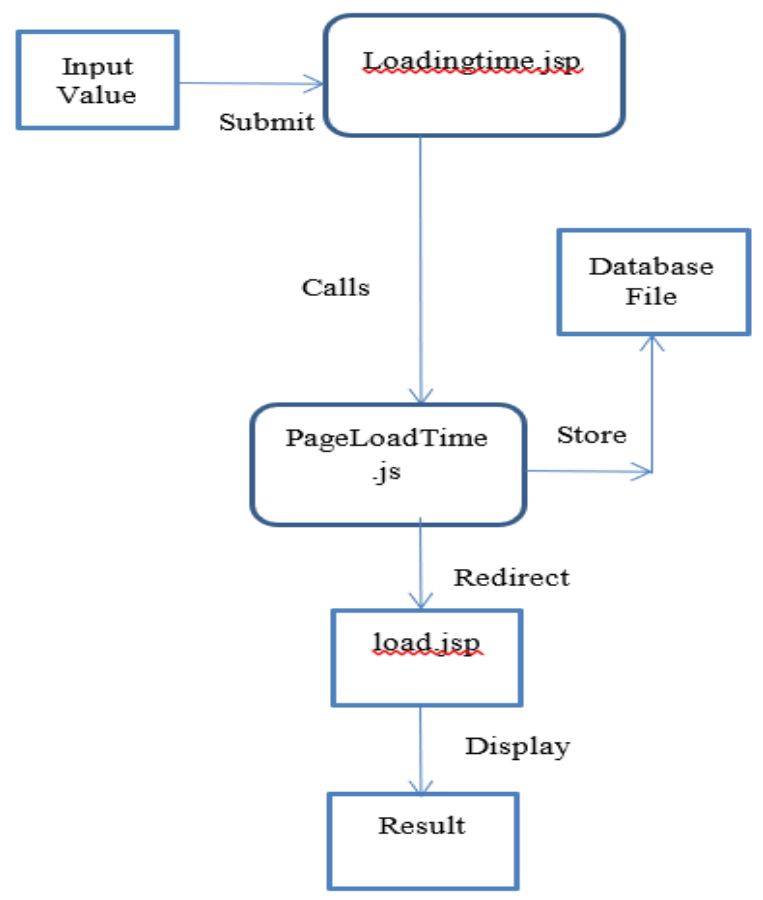

Fig 1: Illustration of Load Testing

\section{RESULT ANALYSIS}

To check the performance of the proposed methodology, I have simulated the method in JAVA on a CPU @ 2.12Ghz and 8GB RAM. The outcome of the simulation is shown in below figure 2 .

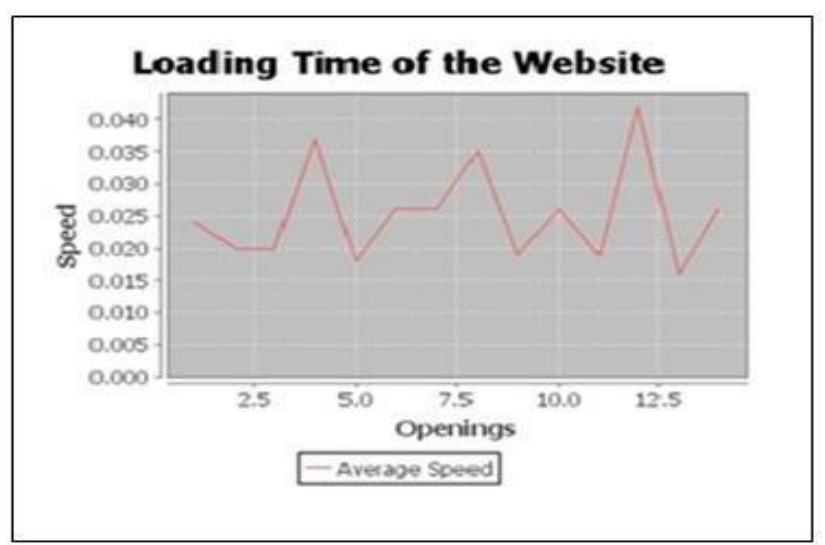

Fig 2: The illustration of the Load Time. 
The directly above indicated depiction directly produced from the database table contains date, opening, time attributes. The number of times the clients hit online application page, every time the data is stored in the database.

A large variety of individuals use the web applications and they are on expanding requests because of the web innovation. To achieve the good performance the testing is done and to guarantee that the online site is dependable. The database collects the information because of the easiness of the stress testing and the pinnacle point can be examined whenever and the shortcomings can be found out and revised on time immediately. Along these lines the electronic application holds its picture.

\section{CONCLUSIONS}

Since, web applications are now at the top place due to incorporation of the web innovation. Therefore, due to this unpredictability arises because of online applications, there is enormous client's requests and the methodologies are supposed to meet their necessities. This paper discusses novel method of testing for web-based application and simulation results shows the efficacy of the same. Subsequently, there is high demand of web-based application testing which further, proved as better source while improving the quality and execution of the same.

\section{REFERENCES}

[1] Charu Babbar and Neha Bajpai, "Web Application Performance Analysis based on Component Load Testing," International Journal of Technology and Applied Science, vol. 2, pp. 22-28, 2011.

[2] Giuseppe A. Di Lucca and Anna Rita Fasolino, "Testing Web-based applications: The state of the art and future trends," in Proceedings of the Twenty-ninth Annual International Conference on Computer Software and Applications, vol. 2, pp. 65-69, July 2005.

[3] Mats Grindal, Jeff Offutt and Sten F. Andler, "Combination of Testing Strategies: A survey," Journal of Software Testing, Verification, and Reliability, vol. 15, pp. 167-199, November 2004.

[4] Chien-Hung Liu, Chih-Tung Hsu et al., "Object-Based Data Flow Testing of Web Applications," in Proceedings of the First Asia-Pacific Conference on Quality Software, pp. 7-16, October 2000.

[5] Giuseppe A. Di Lucca and Massimiliano Di Penta, "Considering Browser Interaction in Web Application Testing ," in Proceedings of the Fifth IEEE International Workshop on Website Evolution, pp. 74-81, September 2003.

[6] Alessandro Marchetto, Paolo Tonella and Filippo Ricca, "State-Based Testing of AjaxWeb Applications," in First International Conference on Software Testing, Verification, and Validation, pp. 121-130, April 2008.
[7] David Bainbridge, Ian H. Witten et al., "Stress-Testing General Purpose Digital Library Software," SpringerVerlag, vol. 5714, pp. 203-214, 2009.

[8] Rudolf Ramler, Edgar Weippl et al., "A Quality-Driven Approach to Web Testing," in Proceedings of the International Conference on Web Engineering, 2002.

[9] Antonio Garcia-Dominguez1 · Konstantinos Barmpis et al., "Stress-testing remote model querying APIs for relational and graph-based stores" in Springer, pp. 1047-1075, 2017.

[10] Jeff Tian, Li Ma et al., "A Hierarchical Strategy for Testing Web-Based Applications and Ensuring their Reliability," in Proceedings of Twenty-seventh Annual International Computer Software and Applications Conference, pp. 702-707, November 2003.

[11] Lu Luo, "Software Testing Techniques," Carnegie Mellon University, School of Computer Science, Class Report for 17-939A, March 2010.

[12] Mahshid Helali Moghadam, Markus Borg et al., "Machine Learning to Guide Performance Testing: An Autonomous Test Framework," in IEEE International Conference on Software Testing, Verification and Validation Workshops (ICSTW), April 2019.

[13]Filippo Ricca and Paolo Tonella, "Analysis and Testing of Web Applications," in Proceedings of the Twenty- third International Conference on Software Engineering, pp. 25-34, 2001.

[14] S Pradeep and Yogesh Kumar Sharma, "A Pragmatic Evaluation of Stress and Performance Testing Technologies for Web Based Applications," Amity International Conference on Artificial Intelligence (AICAI), January 2020.

[15]Vineta Arnicane, "Complexity of Equivalence Class and Boundary Value Testing Methods," International Journal of Computer Science and Information Technology, vol. 751, pp. 80-101, 2009.

[16] Alain Abran, Pierre Bourque, Robert Dupuis, James W. Moore \& Leonard L. Tripp (2004): Guide to the Software Engineering Body of Knowledge SWEBOK, 2004 version edition. IEEE Press, Piscataway, NJ, USA.

[17] Boris Beizer (1990): Software testing techniques (2nd ed.). Van Nostrand Reinhold Co., New York, NY, USA.

[18] C. Kallepalli and J. Tian, "Measuring and modeling usage and reliability for statistical web testing", IEEE Transaction on Software Engineering, 27(11): 1023-1036, November, 2001.

\section{ABOUT THE AUTHORS}

Shivangi Kaushal working as an assistant professor at Amity University Haryana. She has completed her B.Tech and M.Tech in Computer Science in 2009 and 2012 respectively. Her research interests include software testing, machine learning and algorithms. 